\title{
Das Projekt der Euro-Rettung und die Widersprüche des europäischen Krisenkonstitutionalismus
}

\begin{abstract}
Die Entwicklung der Europäischen Union, insbesondere der Eurozone, war in den vergangenen Jahren durch krisenkonstitutionalistische Reformprozesse gekennzeichnet. Um zu begreifen, wie sich diese Reformen in den bisherigen Verlauf der europäischen Integration einfügen, wird eine neo-gramscianisch und regulationstheoretisch inspirierte politökonomische Perspektive entwickelt, die die Integrationsgeschichte als einen kriseninduzierten Prozess ausgehandelter Übereinkommen konzeptualisiert. Im Unterschied zu früheren Krisen und hierauf bezogenen politischen Reaktionen stellt sich die aktuelle Konstellation aber spezifisch dar: Zum einen scheint es sich bei der Euro- und Staatsschuldenkrise nicht um eine Entwicklungs-, sondern durchaus um eine Existenzkrise - zumindest der Eurozone - zu handeln, deren Bewältigung höchst ungewiss ist; und zum anderen generiert der europäische Krisenkonstitutionalismus bedenkliche sozioökonomische, demokratiepolitische und zwischenstaatliche Konsequenzen.
\end{abstract}

\section{Einleitung}

Nach über fünf Jahren gibt der Verlauf der europäischen Krisenprozesse noch immer neue Rätsel auf. Dies liegt nicht zuletzt daran, dass sich die Erscheinungsformen der Krise im Zeitablauf wiederholt verändert - erweitert und verlagert - haben. Was als Subprimekrise in den USA begann, entwickelte sich rasch zur Weltfinanz-, Weltwirtschafts-, Staatsschulden- und Eurokrise; und vermittelt über das Krisenmanagement kann in vielen Ländern inzwischen längst von einer tiefen Krise der Arbeitsmärkte, Wohlfahrtsstaaten und demokratischen Systeme gesprochen werden. Doch nach wie vor bleibt unklar, wohin sich die Krise - gerade auch in der EU - weiter bewegt und durch welche Schritte sie überwunden werden kann.

Nachfolgend soll dargelegt werden, wie sich die europäische Krisendynamik aus der Perspektive einer kritischen, neo-gramscianisch und regulationstheoretisch inspirierten Politischen Ökonomie darstellt. In diesem Sinne werden zunächst die Umrisse einer solchen Perspektive rekonstruiert. Der Integrationsprozess wird dabei als eine Abfolge kriseninduzierter, durch die gesellschaftlichen Macht- und Herrschaftsverhältnisse geprägter politischer Projekte konzeptualisiert (2). In einem nächsten Schritt wird dann ausgeführt, dass sich im Laufe der Krise mit der EuroRettung ein neues politisches Projekt in den Vordergrund geschoben hat, das sich allerdings aus unterschiedlichen Initiativen mit zum Teil widersprüchlichen Zielen zusammensetzt (3). Im Kern verdichten sich diese Initiativen in einem europäischen Krisenkonstitutionalismus, d.h. einer partiellen Fortentwicklung supranatio- 
naler Verfassungsbildung und Staatlichkeit (4). Ob die krisenkonstitutionalistischen Reformen ausreichen, die Krisenprozesse zu bewältigen, ist nach wie vor unklar. Weniger unklar ist hingegen, dass der Krisenkonstitutionalismus ernsthafte arbeits-, sozial- und demokratiepolitische sowie integrationspolitische Folgeprobleme provoziert (5).

\section{Krisenprozesse und Integrationsdynamiken}

In der bisherigen Geschichte der europäischen Integration sind Krisen keineswegs ein außergewöhnliches Phänomen. Im Gegenteil, eigentlich lässt sich der Integrationsprozess sehr gut als Krisenprozess rekonstruieren; vor allem dann, wenn man Krisen als Phasen einer - mehr oder minder tiefen - Verunsicherung und Ungewissheit begreift, in denen etablierte Entwicklungspfade erschüttert und aufgrund unvorhergesehener Ereignisse diskursiv in Frage gestellt werden. Krisen sind damit immer auch Zeiträume der Entscheidung und potenziellen Wende, in denen, so Antonio Gramsci (1991: 354) »das Alte stirbt [während] [...] das Neue [...] noch nicht zur Welt kommen [kann] . Die politische Bearbeitung der Verunsicherung und Ungewissheit ist nicht nur durch die unmittelbare Krisenkonstellation bestimmt, sondern auch durch die gesellschaftlichen, vor allem politökonomischen Kontextbedingungen, insbesondere durch konkurrierende Interessenlagen, Machtverhältnisse, Diskurse und Deutungsangebote (Jessop 2009). So können z.B., wenn sich eine weithin geteilte Krisendiagnose - angesichts ähnlicher oder komplementärer Interessenlagen - in ausstrahlungsfähige Initiativen oder Projekte der Krisenbewältigung übersetzt, Krisen mitunter eine produktive Wirkung erzeugen.

In der Geschichte der europäischen Integration lassen sich mehrere Krisenkonstellationen identifizieren, die paradoxerweise nicht zu einem Rückschritt geführt haben, sondern vielmehr - vermittelt über krisenpolitische Diskurse, Projekte und Initiativen - die Integration stabilisiert und dynamisiert haben (vgl. Deppe 1993; Kirt 2001). So hatte das Scheitern der Europäischen Verteidigungsgemeinschaft (EVG) im Jahr 1954 schon kurz darauf die Pariser Verträge und den Nato-Beitritt Westdeutschlands zur Folge; de Gaulles Politik des leeren Stuhls 1965/66 führte nicht nur zum Luxemburger Kompromiss, sondern bald auch zum Gipfel von den Haag, mit dem wichtige integrationspolitische Initiativen wie z.B. eine gemeinsame Finanzverfassung, die Vollendung der Gemeinsamen Agrarpolitik (GAP), die Europäische Politische Zusammenarbeit (EPZ) oder die Norderweiterung eingeleitet wurden; auf das Scheitern des Werner Plans und das Auseinanderbrechen der Währungsschlange in den 1970er Jahren folgte die Gründung des Europäischen Währungssystems (EWS); die Diskussionen über eine sog. Eurosklerose und über einen wachsenden technologischen Rückstand gegenüber den USA und Japan stimulierten das EG-Binnenmarktprojekt und die Einheitliche Europäische Akte (EEA); die Wirtschafts- und Währungsunion (WWU) bildete auch eine Antwort auf die durch die Kapitalmarktliberalisierung generierte Instabilität des EWS und das Problem, das vereinigte Deutschland europäisch einzubinden; und die Finanzmarktintegrati- 
on und die Lissabon-Strategie stellten Projekte dar, mit Hilfe derer die Modernisierung der europäischen Wirtschafts- und Sozialsysteme gemäß den Anforderungen des globalen Finanzmarktkapitalismus vorangetrieben werden sollte.

Die Besonderheiten der genannten Krisenprozesse sollen hier keineswegs in Abrede gestellt werden. Gleichwohl gibt es ein übergreifendes Muster, das für den Verlauf der europäischen Integration charakteristisch zu sein scheint. So lässt sich die kriseninduzierte Generierung europapolitischer Projekte und Initiativen als ein Prozess verstehen, in dem zwischen staatlichen und gesellschaftlichen Akteuren spezifische politische Kompromisse kommunikativ beraten und ausgehandelt werden, die letztlich auf die strukturellen gesellschaftlichen und integrationspolitischen Kontextbedingungen zurückwirken, also die politökonomischen, vertraglichen und politisch-institutionellen Bedingungen der Integration transformieren, zuweilen auf eine neue Stufe heben. Dies schließt auch die legitimationspolitisch zentrale Frage mit ein, ob und in welchem Maße derartige Entwicklungen hegemonial abgesichert werden, d.h. sich auf einen breiten Konsens stützen können. Um die unterschiedlichen Quellen und Kontexte der Konsens- oder aber auch Dissens-Erzeugung einzufangen, kann aus der Perspektive einer neo-gramscianisch und regulationstheoretisch inspirierten Politischen Ökonomie zwischen mehreren, aufeinander bezogenen Analyseebenen differenziert werden:

(1) Die erste Analyseebene bilden die historisch-strukturellen Dimensionen der gesellschaftlichen Entwicklung. In der Regulationstheorie werden diese historischstrukturellen Dimensionen im Begriff des »Entwicklungsmodells« (Lipietz 1985) und im Neo-Gramscianismus in der Konzeption des (transnationalen) »historischen Blocks" (Cox 1983) zusammengeführt und zu charakterisieren versucht. Beide Konzepte befassen sich mit der Frage, wie sich innerhalb einer spezifischen kapitalistischen Gesellschaftsformation unterschiedliche - ökonomische, politische, soziale, kulturelle und diskursive - Dynamiken artikulieren und reproduzieren. Mit Blick auf die europäische Entwicklung seit dem Zweiten Weltkrieg werden zumeist zwei Formationen unterschieden: zum einen die des Fordismus, die in hohem Maße nationalstaatlich organisiert war und durch die trans- und supranationalen Prozesse der Liberalisierung und Integration nur schwach überformt wurde; und zum anderen die Formation des Finanzmarktkapitalismus, die durch eine zentrale Bedeutung der Kapitalmärkte und durch diese geförderte Finanzialisierungsprozesse (vgl. Heires/Nölke 2013) sowie durch ausgeprägte internationale ökonomische Interdependenzen und diese stabilisierende trans- und supranationale Organisationsmuster gekennzeichnet ist. Letzteres verweist auf ein erhöhtes Integrationsniveau, das aufgrund der ungleichen Entwicklung und des asymmetrischen Charakters der ökonomischen Interdependenzen - die Krise hat dies sehr deutlich vor Augen geführt (vgl. Becker/Jäger 2012; Lapavitsas et al. 2012) - zugleich aber neuartige, durch Gläubiger-Schuldner-Beziehungen konstituierte Spannungen und Konflikte erzeugt.

(2) Die politischen Auseinandersetzungen verweisen zugleich auf die zweite Analyseebene der sozialen Beziehungen und Allianzen. Diese werden in der neogramscianischen Internationalen Politischen Ökonomie (IPÖ) wie auch zuweilen in 
regulationstheoretischen Arbeiten als hegemonialer Block bezeichnet; zumindest dann, wenn sich das gesellschaftliche Machtgefüge durch materielle und symbolische Konzessionen, Kompromisse und konsensuale Auffassungen reproduziert, sich also auf die Zustimmung großer Teile der subalternen sozialen Klassen oder Gruppen stützen kann. Bezogen auf die aktuelle integrationspolitische Konstellation ist das Konzept des hegemonialen Blocks allerdings in dreifacher Hinsicht zu spezifizieren (vgl. Macartney 2011). Erstens hat sich die Reproduktion der gesellschaftlichen Machtgefüge unverkennbar transnationalisiert, weshalb auch auf dieser Analyseebene vielleicht besser von einem transnationalen hegemonialen Block die Rede sein sollte; zweitens sind die tonangebenden Kräfte innerhalb dieses Blocks inzwischen vor allem finanzmarktorientierte Akteursgruppen wie institutionelle Anleger, Rating-Agenturen, Groß- und Investmentbanken, transnationale Konzerne, Consultants sowie marktliberale Think Tanks, Politiker und Bürokraten; und drittens wird der hegemoniale Charakter der sozialen und politischen Organisationsmuster zunehmend in Frage gestellt, da im transnationalen Machtblock die - gesellschaftlichen und intergouvernementalen - Widersprüche und Risse zunehmen.

(3) Möglicherweise können diese Risse, die sich innerhalb der nationalen Gesellschaften wie auch zwischen den Regierungen manifestieren, durch neue (europa-)politische Projekte produktiv bearbeitet und gekittet werden. Wie bereits angedeutet, handelt es sich bei politischen Projekten um eine programmatische und politisch-operative Kristallisation spezifischer Interessen und Diskurse, über die gesellschaftspolitische Akteure oder besser vielleicht (transnationale) Allianzen auf den Fortgang der Integration Einfluss nehmen. Wie dies geschieht, ist historisch offen und umkämpft, mithin kontingent. So können die lancierten politischen Projekte dazu beitragen, die tradierten institutionellen und regulativen Arrangements, einschließlich der Machtverhältnisse sowie Kompromiss- und Konsensstrukturen zu stabilisieren. Je nach Krisennarrativ und sozialer Interessenlage können die Projekte - grundsätzlich - aber auch so beschaffen sein, dass sie den eingeschlagenen Entwicklungspfad, die etablierten sozialen Interaktionsmuster und institutionellen Arrangements transformieren oder substanziell in Frage stellen. Die Ausstrahlung und Gestaltungskraft - von nationalen und europäischen - politischen Projekten ist zumeist dann besonders groß, wenn unterschiedliche Diskurs-Ebenen - die Ebene der konzeptiven Intellektuellen und Think Tanks, die Ebene der meinungspolitischen Multiplikatoren sowie die Ebene des fragmentiert-widersprüchlichen Alltagsbewusstseins - gut ineinander greifen und sich wechselseitig bestärken (Hall 1989: $80-84){ }^{1}$

1 Die unterschiedlichen Ebenen des Diskurses verdeutlichen, dass die Ausprägung der politischen Projekte in der praktischen Umsetzung durch unterschiedliche Dimensionen - überlieferte Sichtweisen, Mythen und kulturelle Traditionen, Konzessionen und Kompromisse sowie die geographisch und sozialstrukturell besonderen Alltagserfahrungen - beeinflusst wird. Die Alltagserfahrungen zeigen recht gut, dass die konkurrierenden gesellschafts- und integrationspolitischen Diskurse - wie vermittelt auch immer - an die politökonomischen (Krisen-)Dynamiken rückgebunden bleiben. 
In der Vergangenheit konnten die europäischen Krisenprozesse durch spezifische (integrations-)politische Projekte zumeist relativ rasch und integrationsfördernd bewältigt werden. So gelang es den diskurs- und entscheidungsprägenden Akteuren wiederholt, im Krisenverlauf neue Projekte zu initiieren und durch eine damit verbundene programmatische Zuspitzung das europäische Gemeinschaftsinteresse zu stärken und zu redefinieren. In diesem Sinne handelte es sich bei den vergangenen Krisen nicht um Existenz-, sondern vielmehr um Entwicklungskrisen, d.h. Katalysatoren einer begrenzten Umformung und Weiterentwicklung der regionalen Integrationsdynamik (vgl. hierzu Deppe 1993: 7-14). Ob dies auch auf die jüngeren Krisendynamiken zutrifft, ist allerdings ungewiss. Einiges spricht dafür, dass sich der Krisenverlauf - nach dem Motto: this time is different - den bekannten Mustern entzieht. Nicht nur stellen sich die politökonomischen Erschütterungen als substanziell dar (Schimmelfennig 2012: 395); auch die politischen Reaktionen verdichten sich bislang nur in einem schwachen, defensiven und gesellschaftlich wie intergouvernemental umkämpften, innerhalb der EU demzufolge unterschiedlich definierten Projekt: der Euro-Rettung.

\section{Das politische Projekt der Euro-Rettung}

Obwohl sich die Subprime-Krise in den USA rasch zu einer Finanz- und Wirtschaftskrise mit tendenziell globalen, zumindest transatlantischen Ausmaßen entwickelte, sah es zunächst nicht danach aus, dass die WWU und der Euro hierdurch beeinträchtigt werden würden. Die nationalen Regierungen, die Europäische Kommission und auch die EZB waren in den Jahren 2008 und 2009 vornehmlich damit befasst, den Kollaps europäischer Banken abzuwehren und der wirtschaftlichen Rezession entgegenzuwirken. So wurden in fast allen EU-Staaten spezifische Fonds eingerichtet, um in Bedrängnis geratene Banken durch eine staatliche Rekapitalisierung - Aktienkäufe, Kredite oder Bürgschaften - zu stabilisieren. Außerdem legten die Regierungen in den meisten Ländern beträchtliche Konjunkturprogramme auf, um den ökonomischen Einbruch abzuschwächen (Schelkle 2012). Beide Prozesse wurden von der EZB, die insbesondere auf das wiederholte Austrocknen des Interbankenmarktes reagierte, mit einer deutlich gelockerten, liquiditätssichernden Geldpolitik unterstützt.

Diese Initiativen wie auch die anfangs sehr ambitionierten Vorschläge, die Finanzmärkte zu re-regulieren, lassen sich als partielle Erschütterung und Desorientierung eines - auf Deregulierung, allokationseffiziente Kapitalmärkte oder den Rückzug des Staates vertrauenden - neoliberal mindset interpretieren (Jessop 2009: 350). Sie sind diskursiv unter anderem mit Verweis auf die Erfahrungen aus den 1930er Jahren legitimiert worden. Damals hatten sich die Regierungen nicht auf eine aktive, international abgestimmte Wirtschafts- und Geldpolitik verständigen können, was eine ausgeprägte Fragmentierung der Weltökonomie zur Folge hatte. Dieses Krisennarrativ ermöglichte zunächst die Hinwendung zu einer diskretionärstaatsinterventionistischen Stabilisierungspolitik. Es trat dann aber in dem Maße in 
den Hintergrund, wie sich die Aufmerksamkeit auf die öffentlichen Kosten des Krisenmanagements, also die steigende Staatsverschuldung und die zunehmende Fragilität der WWU konzentrierte (Overbeek 2012: 38-40). Wie sich die Verknüpfung zwischen beiden Phänomenen - der Staatsschuldenkrise und der Euro-Krise - genau darstellt, war in den politökonomischen Diskussionen freilich umstritten: Einige sahen die Krisenursachen bereits im Design der WWU angelegt, das den Anforderungen an eine Optimal Currency Area (OCA) - ökonomische Konvergenz, transnational flexible Arbeits- und Gütermärkte und fiskalische Ausgleichsmechanismen - nicht entsprach (vgl. Scharpf 2011a; de Grauwe 2013). Andere lenkten komplementär zu dieser modelltheoretischen Perspektive den Blick stärker auf die finanzmarktkapitalistische Akkumulationsdynamik, d.h. auf die Instabilität des Finanzsystems, aber auch auf die politisch-konfliktiven Verlaufsformen des Krisenmanagements, die sich unter anderem aus den strukturellen Ungleichgewichten in den innereuropäischen Leistungsbilanzen, also daraus speisten, dass einer Gruppe von Überschuss- und Gläubigerländern eine Gruppe von Defizit- und Schuldnerländern gegenüberstand, die wachsende Schwierigkeiten hatten, die Staatsschulden auf den freien Kapitalmärkten durch neue Kredite zu refinanzieren (vgl. Bieling 2011; Becker/Jäger 2012). Wieder andere machten hingegen in erster Linie eine unsolide Haushaltspolitik der nationalen Regierungen und einen $\mathrm{zu}$ locker definierten europäischen Rahmen der wirtschafts-, finanz- und geldpolitischen Koordinierung für die zunehmenden Spannungen in der EU verantwortlich (vgl. Heinemann et al. 2012; Vaubel 2012).

In dem Maße, wie sich im Laufe des Jahres 2010 die Staatsschuldenkrise - zunächst in Griechenland, dann aber auch in Irland, Portugal, Spanien, Italien und zuletzt Zypern - verschärfte, rückte im politischen Diskurs die letztgenannte Kriseninterpretation in den Vordergrund. Vor allem die Regierungen der sog. nordischen Länder - in der Eurozone: Deutschland, Niederlande, Österreich, Finnland -, deren Volkswirtschaften beachtliche Leistungsbilanzüberschüsse erzielen und deren Finanzinstitute entsprechend eine starke internationale Gläubigerposition einnehmen, trieben diese diskursive Orientierung voran. Sie wurden dabei von der Europäischen Kommission, der EZB und Verbänden der transnationalen Finanz-, Dienstleistungs- und Industriekonzerne unterstützt, denen ebenfalls an einer Begrenzung und Reduktion der öffentlichen Schulden gelegen war (vgl. Bieling 2011; Schimmelfennig 2012: 407). Die Diskussionen über das europäische Krisenmanagement und die konkret ausgehandelten Schritte waren dabei durch gewisse Engführungen gekennzeichnet: So wurden die Leistungsbilanzungleichgewichte relativ einseitig als Problem einer unzureichenden Wettbewerbsfähigkeit der Defizitländer, die ihre Hausaufgaben nicht gemacht hätten, diskutiert; zugleich wurde die in vielen Ländern sehr hohe private Schuldenlast und Fragilität der Kreditsysteme, also die vermehrte Blasen-Entwicklung im Finanzmarktkapitalismus, kaum als Krisenursache thematisiert; stattdessen kam es zu einer recht unmittelbaren Verknüpfung von Staatsschuldenkrise und Euro-Krise; und da es letztere - mit Verweis auf die mutmaßlich beträchtlichen, präzise aber kaum kalkulierbaren Kosten einer Auflösung 
der WWU - unbedingt abzuwenden galt, gewannen im Umkehrschluss austeritätspolitische Konzeptionen an Bedeutung. ${ }^{2}$

Diese diskursive Fokussierung, aber auch die Interessen der Überschussländer und Geldvermögensbesitzer machen verständlich, warum das politische Projekt der Euro-Rettung, das seit 2010 die europäische Agenda bestimmt, austeritätspolitisch zugeschnitten wurde. Zugleich ist zu berücksichtigen, dass dieser Zuschnitt im Aushandlungsprozess durch einige Konzessionen und Spezifizierungen modifiziert worden ist. Vor allem zwei Aspekte spielten eine wichtige Rolle:

Der eine Aspekt besteht darin, dass der politische Diskurs der Gläubigerstaaten nicht nur durch politökonomisch gleiche oder zumindest ähnliche Interessenlagen bestimmt ist, sondern auch durch spezifische wirtschaftspolitische Kulturen. In Deutschland zeigt sich dies in der wiederholten, zum Teil sogar sehr expliziten, Bezugnahme auf das ordoliberale Konzept einer Regeln setzenden Politik, die jedoch möglichst auf unmittelbare staatliche Interventionen verzichtet (vgl. Scharpf 2011b; Young 2013). Was dies bedeutet, hat unter anderem die deutsche Bundeskanzlerin wiederholt zum Ausdruck gebracht, so z.B. in einer Regierungserklärung vom 2. November 2011:

\begin{abstract}
»Weil die gegenwärtige Krise im Euro-Raum vor allem eine Vertrauenskrise ist, müssen wir neben der Bekämpfung der Ursachen dieser Krise - zu hohe Staatsverschuldung, mangelnde Wettbewerbsfähigkeit einiger Euro-Staaten - die grundlegenden Mängel in der Konstruktion der Wirtschafts- und Währungsunion beseitigen. Wenn wir das machen, dann zeigen wir im Übrigen, dass wir nicht nur die Mühen der Krise sehen, sondern diese Krise vor allem als eine Wende zum Guten, als eine Chance zur Umkehr begreifen und dass wir tatsächlich aus ihr lernen. Das sind ja ganz einfache Lehren: Regeln müssen eingehalten werden; ihre Einhaltung muss kontrolliert werden; ihre Nichteinhaltung muss Konsequenzen haben. Nationale Eigenverantwortung und europäische Solidarität bedingen einander « (Merkel 2011).
\end{abstract}

Die Regierungen der nordischen Länder und private Gläubiger haben die disziplinierenden ordnungspolitischen Impulse in den diversen Initiativen zur Euro-Rettung wiederholt zu stärken versucht. Ebenso mussten in den Verhandlungen über das europäische Krisenmanagement - und dies ist der zweite, leicht gegenläufige Aspekt - gewisse Konzessionen gemacht werden. Zum einen wurden die Interessen der Defizitländer insofern berücksichtigt, als diesen über die Einrichtung eines europäischen Rettungsfonds, zunächst der European Financial Stability Facility (EFSF), inzwischen des European Stability Mechanism (ESM), und den unbeschränkten Aufkauf von Staatsanleihen durch die EZB gemeinschaftliche Ressourcen zur Verfügung gestellt wurden, um die finanzpolitisch disziplinierende Reformagenda zeitlich zu strecken und punktuell etwas zu entschärfen. Wenn in diesen Instrumenten zuweilen der Einstieg in eine Transferunion gesehen wird, so ist dies allerdings insofern etwas irreführend, als die Gläubigerländer ihrerseits von

2 Grundsätzlich wäre es auch möglich gewesen, eine haushaltspolitische Konsolidierung auf dem Wege eines höheren Wirtschaftswachstums mit entsprechenden Steuermehreinnahmen anzustreben. Da eine solche Strategie für die wirtschaftlich starken Länder zunächst aber beträchtliche Kosten mit sich bringt und die öffentlichen Defizite zumindest kurzfristig vergrößert, erschien sie den politischen Entscheidungsträgern als zu riskant. 
übermäßig reduzierten Zinssätzen auf Staatspapiere profitierten, und die den Schuldnerstaaten gewährten Gemeinschafts-Kredite in beträchtlichem Umfang und mehr oder minder umgehend an die Banken aus den Gläubigerstaaten zurückflossen. Zum anderen waren der Rettungsfonds, die Interventionen der EZB wie auch die wiederholten Schritte, die Funktionsweise der WWU zu reformieren, zugleich auch Konzessionen an eine höchst unsichere, existenziell bedrohliche Krisenkonstellation. Die europäischen Entscheidungsträger zeigten sich zum Teil bereit, so z.B. im Fall von EFSF/ESM, EZB-Interventionen oder Fiskalvertrag (Joerges 2012: 365-376), vertragsrechtlich bedenkliche Initiativen zu ergreifen, um eine Existenzkrise der WWU und vielleicht sogar der gesamten EU abzuwehren oder diese zumindest in eine Entwicklungskrise zu überführen, in der der Integrationsprozess durch neue gemeinsame Reformprojekte stabilisiert, vertieft und vorangebracht werden kann.

\section{Elemente und Facetten des europäischen Krisenkonstitutionalismus}

$\mathrm{Ob}$ mit den genannten Initiativen das angestrebte Ziel erreicht wird, ist angesichts der fortdauernden Krisenprozesse und ökonomischen Ungleichgewichte noch immer ungewiss und zweifelhaft. Die dichte Abfolge von Krisengipfeln, auf denen jeweils spezifische Maßnahmen und Reformschritte ausgehandelt wurden, verweist jedoch auf ein aktives Krisenmanagement. Die inkrementell-kaskadenförmige Neujustierung der Funktionsbedingungen der WWU ist inzwischen relativ umfassend. Daher werden die wichtigsten Änderungen hier nur knapp resümiert: Erstens kam es zu der bereits erwähnten ad hoc Stabilisierung der WWU durch interventionistische Instrumente wie die EFSF und den ESM oder die pragmatische Geldpolitik der EZB. Zweitens versuchten die Regierungen, die Kommission und viele zivilgesellschaftliche Akteure, die beträchtlichen Schlupflöcher in der europäischen - und auch globalen - Finanzmarktregulierung - zu schließen; ein Prozess der anfangs sehr ambitioniert gestartet war, in vielen Punkten bislang aber nur bescheidene Ergebnisse zeitigte (zum Überblick vgl. Troost 2011; Quaglia 2012). Drittens schließlich konzentrierten sich die europäischen Aktivitäten zunehmend darauf, die nationalen wirtschafts- und finanzpolitischen Prozesse, die auf die Funktionsweise der WWU zurückwirken, verbindlicher und präskriptiver zu definieren (vgl. Hacker/van Treeck 2010; Konecny 2012).

So gibt es inzwischen ein Europäisches Semester, durch das die Europäische Kommission und der Europäische Rat versuchen, präventiv auf die Struktur und die politischen Prioritäten der nationalen Haushalte Einfluss zu nehmen. Zudem wurde ein sog. sixpack verabschiedet, d.h. ein Gesetzespaket aus fünf Verordnungen und einer Richtlinie, um den Stabilitäts- und Wachstumspakt (SWP) zu reformieren. Zukünftig werden zumindest partiell, allerdings stark asymmetrisch, auch Leistungsbilanzungleichgewichte berücksichtigt, vor allem aber können Sanktionen im Fall einer Nichtbefolgung nur noch durch Zweidrittel-Mehrheiten abgewehrt werden. In Ergänzung zum reformierten SWP wurde außerdem ein Euro-plus-Pakt 
ausgehandelt (Gros/Alcidi 2011), der gestützt auf ein Selbstverpflichtungs-Verfahren nachdrücklich auf eine wettbewerbsorientierte Lohn-, Arbeitsmarkt-, Sozialund Steuerpolitik hinwirken soll. Und zuletzt verständigten sich die Regierungen zudem auf einen Fiskalvertrag und eine Bankenunion. Während sich die beteiligten Regierungen mit dem Fiskalvertrag verpflichten, die europäischen haushaltspolitischen Vorgaben in ihren Verfassungen festzuschreiben, bringt die Bankenunion eine bei der EZB zentralisierte Bankenaufsicht, ein grenzüberschreitendes Einlagensicherungssystem sowie direkt über den ESM laufende Verfahren der Bankenrettung mit sich.

Zusätzlich zu den aufgeführten Aktivitäten gibt es weitere, bislang nur diskutierte oder noch nicht umgesetzte Initiativen und Überlegungen zur Reform der WWU. Diese reichen von einer gemeinschaftlichen Schuldenhaftung bis hin zu weiteren Elementen einer intensivierten wirtschaftspolitischen Koordination und haushaltspolitischen Überwachung (Europäischer Rat 2012). Doch selbst wenn diese Überlegungen nicht realisiert werden, sollte erkennbar geworden sein, dass die europäischen Reformaktivitäten die wirtschafts- und gesellschaftspolitischen Organisationsprozesse zum Teil substanziell verändern. Es ist genau dieser substanzielle Charakter und die erschwerte Revidierbarkeit der ausgehandelten institutionellen und rechtlichen Übereinkünfte - zumeist sind einstimmige oder qualifizierte Mehrheitsentscheidungen erforderlich -, die es rechtfertigen, die genannten Reformschritte als Elemente einer impliziten europäischen Verfassungspolitik, d.h. einer krisenkonstitutionalistischen Fortentwicklung der gemeinschaftlichen institutionellen und regulativen Arrangements, zu betrachten. ${ }^{3}$

Der Krisenkonstitutionalismus repräsentiert mithin eine neue, die nationalen wirtschafts- und finanzpolitischen Handlungsmöglichkeiten vielfach restringierende Form europäischer Verfassungspolitik, die sich von früheren Phasen klar unterscheidet. Die Differenz ist besonders deutlich im Verhältnis zum alten Konstitutionalismus der Römischen Verträge, der im Sinne eines »embedded liberalism« (Ruggie 1982) die nationalen Entwicklungsmodelle durch eine begrenzte und wachstumsstimulierende Liberalisierung förderte und abstützte. Etwas weniger klar und eindeutig ist hingegen die Abgrenzung vom neuen Konstitutionalismus der zurückliegenden Jahrzehnte. Dieser zielte in erster Linie darauf, die markt- und wettbewerbsorientierten Reorganisationsprozesse rechtlich und institutionell abzusichern, den Modernisierungs- und Anpassungsdruck auf die nationalen Entwicklungsmodelle mithin zu erhöhen, und die europäischen Vorgaben zugleich einer zukünftigen demokratischen Kontrolle und Gestaltung zu entziehen (Gill 1998). Durch den europäischen Krisenkonstitutionalismus werden die - wirtschafts- und finanzpolitisch - disziplinierenden Elemente der gemeinschaftlichen Rechts- und Institutionenordnung nochmals gestärkt. Von daher gibt es eine deutliche Kontinui-

3 Das hier entfaltete politökonomische Verständnis internationaler Verfassungsbildung mag etwas irritieren, da es im Kontrast zur rechtswissenschaftlichen Perspektive, die vor allem die normativen Geltungsgrundlagen des Verfassungsrechts beleuchtet, umfassender angelegt ist und sich auf die institutionellen, regulativen und rechtlichen Dimensionen einer entstehenden europäischen Gesellschaftsverfassung bezieht (vgl. auch Eberl/Rödl 2010). 
tätslinie. Zugleich markiert der Krisenkonstitutionalismus aber auch insofern einen Bruch, als er sich nicht einfach darauf beschränkt, die systemischen Vorgaben der (Finanzmarkt-)Globalisierung technokratisch zu flankieren. Im Zeichen eines »permanent economic emergency« (Žižek 2010) sind eine Vielzahl neuer Interventionsinstrumente geschaffen worden, um die Instabilität und die Krisenanfälligkeit des europäischen Finanzmarktkapitalismus zu bearbeiten. Im Vergleich zum neuen Konstitutionalismus ist der Krisenkonstitutionalismus demzufolge sehr viel stärker politisiert. Offenkundig ringen die politischen Akteure sehr hart um die mutmaßlich angemessenen Strategien und Maßnahmen, die einen nachhaltigen und aus ihrer Sicht relativ kostengünstigen Weg aus der Krise weisen sollen.

Dass es bislang nicht so recht gelungen ist, die widerstreitenden Perspektiven auszutarieren und das politische Projekt der Euro-Rettung als gemeinwohlorientiert und allseits akzeptierbar zu präsentieren, hat mehrere Ursachen. Eine allgemeine Ursache besteht in den strukturellen Schwierigkeiten, die globalisierten Finanzmärkte effektiv zu re-regulieren und unter politische Kontrolle zu bringen (Kessler 2009). Die hiermit verbundenen regulativen, letztlich aber auch macht- und verteilungspolitischen Konflikte durchziehen - mehr oder minder - alle Gesellschaften. Darüber hinaus wird der europäische Finanzmarktkapitalismus vor allem durch eine politisch fehlkonstruierte WWU belastet. Die Konstruktionsfehler bestehen darin, dass unter den Bedingungen einer einheitlichen Geldpolitik - eines gemeinsamen Leitzinses für alle - und aufgrund des Wegfalls von Wechselkursanpassungen eigentlich zusätzliche gemeinsame Instrumente und Ressourcen, unter Einschluss erweiterter wirtschafts-, industrie- und transferpolitischer Kompetenzen, erforderlich wären, um die ungleichgewichtige Entwicklung in der Eurozone auszubalancieren. Wie dargestellt, wurden Vorstöße in diese Richtung seitens der Leistungsbilanzüberschussländer jedoch weitgehend zurückgewiesen, so dass die internen Ungleichgewichte zunahmen und ihrerseits die europäische Krisendynamik verschärft haben. Nach wie vor stützt sich die WWU allenfalls auf eine fragmentierte politische Souveränität; mit der Folge, dass die lender of last resort-Funktion - zwischen den nationalen Regierungen sowie zwischen den Regierungen und der EZB - ungeklärt und umkämpft bleibt. Mehr noch, aufgrund der bestehenden Machtverhältnisse stellt sich die WWU für die wirtschaftlich schwächeren Länder als ein Regime der Fremdkontrolle dar. Von Deutschland einmal abgesehen, so Michel Aglietta, repräsentiert der Euro:

$»[\ldots]$ essentially a foreign currency for every Eurozone country. It binds them to rigidly fixed exchange rates, regardless of their underlying economic realities, and strips them of monetary autonomy« (Aglietta 2012: 20).

Auch die krisenkonstitutionalistischen Reformschritte dürften nur wenig dazu beitragen, die Operationsweise der WWU auf eine breitere, auf sozialen Ausgleich und gleichberechtigte Teilhabe basierende, Grundlage zu stellen. Eher zeichnet sich das Gegenteil ab. Vor allem zwei Entwicklungen stimmen bedenklich. Zum einen ist der Zeitgewinn, der durch die diversen Maßnahmen einer kredit- und geldpolitischen ad hoc-Stabilisierung vorübergehend generiert wurde, bislang vornehmlich dazu genutzt worden, das europäische Disziplinierungs- und Kontrollregime, nicht 
aber die wirtschafts- und sozialpolitischen Handlungskapazitäten in den Krisenländern zu stärken; und zum anderen sind die asymmetrischen Beziehungen zwischen dem europäischen Machtzentrum - Deutschland, Frankreich und Länder mit Leistungsbilanzüberschüssen - und der süd- und osteuropäischen Peripherie, die in der Vergangenheit kooperativ abgemildert wurden, im Krisenverlauf deutlich stärker hervorgetreten (Beck 2012: 40-45; Offe 2013).

\section{Krisenkonstitutionalistische Folgeprobleme und Widersprüche}

Die zuletzt genannten Prozesse weisen bereits auf einige brisante Implikationen des europäischen Krisenkonstitutionalismus hin. Diese ergeben sich zum Teil daraus, dass sich die Reformprozesse primär auf einen europäischen »Exekutivföderalismus«, d.h. die »intergouvernementale Herrschaft des Europäischen Rates« stützen und dieser - im Zeichen der Krise - verstärkt dazu neigt, intransparente und »rechtlich formlose Vereinbarungen« zu treffen, die »Imperative der Märkte an die nationalen Haushalte « (Habermas 2011: 81) weiterzugeben und die nationalen Parlamente zu entmachten. Darüber hinaus erzeugt der Krisenkonstitutionalismus aber auch deswegen gravierende Folgeprobleme, weil er im Kern die Operationsweise der WWU fortschreibt, dabei aber zugleich die Disziplinierung der nationalen Finanz-, Wirtschafts- sowie der Arbeits- und Sozialpolitik nochmals verschärft. In der Konsequenz wird hierdurch in der EU eine austeritätspolitische Agenda verallgemeinert, die einer ökonomischen Erholung entgegensteht und in der europäischen Peripherie einen circulus vitiosus aus öffentlichen Ausgabenkürzungen, stagnierendem oder rückläufigem Wirtschaftswachstum, schrumpfenden Steuereinnahmen und hohen öffentlichen Haushaltsdefiziten etabliert. In der Verbindung dieser beiden Dimensionen - der exekutivföderalistischen Kontrolle und austeritätspolitischen Disziplinierung - entstehen letztlich weitere Krisenprozesse, die sich wechselseitig verstärken und den Fortgang der europäischen Integration schwer belasten:

Als erstes ist auf die beschleunigte Erosion der sozialen Grundlagen und Bindungen zu verweisen. Vor allem in den Gesellschaften, die sehr hart von der Krise erfasst wurden und die gemäß der austeritätspolitischen Auflagen der Troika - bestehend aus EU-Kommission, IWF und EZB - ihre Wettbewerbsfähigkeit durch eine sog. interne Abwertung, d.h. eine arbeits- und sozialpolitische Kostenreduktion, verbessern sollen, stellt sich die soziale Lage dramatisch dar. Aber auch in anderen Ländern zielen die Konsolidierungsprogramme überwiegend darauf, die öffentlichen Ausgaben durch soziale Einschnitte - diese betreffen öffentliche Leistungen und Investitionen, die Beschäftigungsbedingungen im öffentlichen Sektor (Arbeitsplatzabbau, Lohnkürzungen) oder die Rentensysteme (Privatisierung, längere effektive Lebensarbeitszeit) - zu reduzieren (vgl. Heise/Lierse 2011; Busch et al. 2012). Sofern zusätzliche Einnahmen durch höhere Steuern generiert werden sollen, handelt es sich zumeist um indirekte Steuern, was ebenfalls eine sozial regressive Verteilungswirkung mit sich bringt. Hinzu kommt die hohe Erwerbslosigkeit, vor allem von jüngeren, zum Teil sehr gut qualifizierten Personengruppen (Chung et al. 
2012). Die damit verbundene Entwertung der beruflichen Qualifikationen ist nicht nur wirtschaftlich besorgniserregend. Sie nährt auch vielfältige Kränkungen, Frustrationen und Zukunftsängste, die es gerechtfertigt erscheinen lassen, von einer verfestigten sozialen Krise zu sprechen.

Wie bereits angedeutet, verschärft der Krisenkonstitutionalismus zweitens auch die Krise demokratischer Diskussions- und Entscheidungsprozesse. Über diese wird seit einiger Zeit bereits unter dem Label »Postdemokratie« (Crouch 2008), d.h. mit Blick auf den voranschreitenden Rückzug der Massen aus der Politik, diskutiert. Durch das europäische Krisenmanagement werden derartige Tendenzen zunächst begünstigt. So breiten sich in vielen Mitgliedstaaten die sozialen Ungleichheiten und Unsicherheiten und damit auch politisch-resignative Stimmungslagen weiter aus. Außerdem haben sich nach einer kurzen ideologisch-diskursiven Erschütterung die transnationalen finanzkapitalistischen Allianzen wieder einigermaßen stabilisiert (Heinrich 2012), indessen die partielle Entmachtung des Europäischen Parlaments (EP) und der nationalen Parlamente voranschreitet. Zugleich kommt es aber auch zu gegenläufigen Entwicklungen. So können, wie die Wahlniederlage von Monti in Italien zeigt, die Repräsentanten der finanzmarktkapitalistischen Allianzen oft nur noch eine sehr begrenzte öffentliche Unterstützung erwarten. Außerdem verweisen die Proteste, Demonstrationen und mitunter sogar grenzüberschreitend organisierten Streiks auf eine erneute, massenbasierte Politisierung europäischer Entwicklungen (Hamman et al. 2012).

Die soziale und die demokratische Krise überlagern oder verdichten sich drittens in zunehmenden legitimationspolitischen Krisenprozessen, die auch auf die europäische Integration selbst zurückwirken. Dieses Phänomen ist keineswegs gänzlich neu, wie die Diskussionen über die Erosion des permissiven Konsensus, also der stillschweigend-passiven Unterstützung europapolitischer Entwicklungen durch den übergroßen Teil der Bevölkerung, belegen. Im Vergleich zu den 1990er Jahren, in denen bereits von einer »Post-Maastricht-Krise« die Rede war (Deppe 1993), ist der Legitimationsverlust der letzten Jahre jedoch geradezu dramatisch. So ist die Zustimmung zur EU vom Frühjahr 2007 bis Ende 2012 um über 20\%-Punkte von $52 \%$ auf $30 \%$ gefallen, indessen die Ablehnung im gleichen Zeitraum von $15 \%$ auf 29\% anstieg (Eurobarometer 2012: 14). Doch nicht nur diese allgemeinen Trends verweisen auf stürmische Zeiten für den Integrationsprozess; mindestens ebenso wichtig ist, dass sich die Delegitimation aus sehr konträren Quellen speist (Offe 2013: 70-72). Auf der einen Seite stehen die Gläubiger in den Überschussländern, die zunehmend weniger bereit sind, mit weiteren Krediten oder öffentlichen Garantien für die Defizit- und Schuldnerländer einzustehen; und auf der anderen Seite klagen die konditionalisierten Schuldner über die zunehmende Fremdbestimmung und das externe Spardiktat, d.h. die harten und rigiden austeritätspolitischen Auflagen.

Diese Interessenkonstellation generiert notgedrungen zwischenstaatliche Spannungen und Konflikte, die sich im Sinne eines negativen spill-over zuweilen auch man denke nur auf die Verhandlungen über den EU-Haushalt - auf andere Bereiche des europapolitischen Tagesgeschäfts übertragen. Die Gefahr, dass die Re-Nationa- 
lisierung der gesellschaftlichen Solidaritätshorizonte und Diskurse auch die europäische Politik erfasst, scheint nicht unbegründet; zumal das politische Projekt der Euro-Rettung und der hierauf bezogene Krisenkonstitutionalismus deutlich gemacht haben, dass sich die - ehemals - hegemonialen Kräfte in der EU derzeit schwer tun, das übergeordnete europäische Gemeinschaftsinteresse nicht nur abstrakt zu postulieren, sondern zugleich auch durch tragfähige, breit akzeptierte Kompromisse zu unterfüttern und praktisch umzusetzen.

\section{Literatur}

Aglietta, Michel 2012: The European Vortex, in: New Left Review 75, 15-36.

Beck, Ulrich 2012: Das deutsche Europa, Frankfurt a.M.

Becker, Joachim/Jäger, Johannes 2012: Integration in Crisis: A Regulationist Perspective on the Interaction of European Varieties of Capitalism, in: Competition \& Change 16: 3, 169-187.

Bieling, Hans-Jürgen 2011: Vom Krisenmanagement zur neuen Konsolidierungsagenda der EU, in: Prokla. Zeitschrift für kritische Sozialwissenschaft 41: 2, 173-194.

Busch, Klaus/Hermann, Christoph/Hinrichs, Karl/Schulten, Thorsten 2012: Eurokrise, Austeritätspolitik und das Europäische Sozialmodell. Wie die Krisenpolitik in Südeuropa die soziale Dimension der EU bedroht (FES: Internationale Politikanalyse, November 2012).

Chung, Heejung/Bekker, Sonja/Houwing, Hester 2012: Young People and the Post-Recession Labour Market in the Context of Europe 2020, in: Transfer: European Review of Labour and Research 18: 3, 301-317.

Cox, Robert W. 1983: Gramsci, Hegemony and International Relations: An Essay in Method, in: Millennium 12: 2, 162-175.

Crouch, Colin 2008: Postdemokratie, Frankfurt a.M.

De Grauwe, Paul 2013: The Political Economy of the Euro, in: Annual Review of Political Science 16: 9, 1-9, http://www.annualreviews.org/doi/pdf/10.1146/annurev-polisci-060911-085923; 9.3.2013.

Deppe, Frank 1993: Von der »Europhorie« zur Erosion. Anmerkungen zur Post-MaastrichtKrise der EG, in: Deppe, Frank/Felder, Michael: Zur Post-Maastricht Krise der Europäischen Gemeinschaft (EG) (FEG-Arbeitspapier Nr. 10), Marburg, 7-62.

Eberl, Oliver/Rödl, Florian 2010: Kritische Politische Ökonomie und radikale Demokratietheorie. Eine Begegnung auf der Suche nach der postneoliberalen Weltrechtsordnung, in: Kritische Justiz 43: 4, 419-433.

Eurobarometer 2012: Die öffentliche Meinung in der Europäischen Union (Standard Eurobarometer 78, Herbst 2012), Luxemburg, Europäische Kommission.

Europäischer Rat 2012: Auf dem Weg zu einer echten Wirtschafts- und Währungsunion (Bericht des Präsidenten des Europäischen Rates Herman Van Rompuy, Brüssel, 26. Juni 2012, EUCO 120/12).

Gill, Stephen 1998: European Governance and New Constitutionalism: Economic and Monetary Union and Alternatives to Disciplinary Neoliberalism in Europe, in: New Political Economy 3: 1, 5-26.

Gramsci, Antonio 1991: Gefängnishefte, Hamburg.

Gros, Daniel/Alcidi, Cinzia 2011: Was bringt der »Euro-plus-Pakt «?, in: Integration 34: 2, 164-171.

Habermas, Jürgen 2011: Zur Verfassung Europas. Ein Essay, Frankfurt a.M.

Hacker, Björn/van Treeck, Till 2010: What Influence for European Governance? The Reformed Stability and Growth Pact, the Europe 2020 Strategy and the »European Semester« 
(International Policy Analysis, Friedrich Ebert Stiftung), in: http://library.fes.de/pdffiles/id/ipa/07724.pdf: 24.4.2013.

Hall, Stuart 1989: Antonio Gramscis Erneuerung des Marxismus und ihre Bedeutung für die Erforschung von »Rasse« und Ethnizität, in: Hall, Stuart: Ausgewählte Schriften. Ideologie, Kultur, Medien, Neue Rechte, Rassismus, Hamburg, 56-91.

Hamann, Kerstin/Johnston, Alison/Kelly, John 2012: Generalstreiks in Westeuropa 1980-2011, in: Gallas, Alexander/Nowak, Jörg/Wilde, Florian (Hrsg.): Politische Streiks im Europa der Krise, Hamburg, 107-113.

Heinemann, Friedrich/Moessinger, Marc-Daniel/Osterloh, Steffen 2012: Feigenblatt oder fiskalische Zeitenwende? Zur potenziellen Wirksamkeit des Fiskalvertrags, in: Integration 35: 3, 167-182.

Heinrich, Mathis 2012: Zwischen Bankenrettungen und autoritärem Wettbewerbsregime. Zur Dynamik des europäischen Krisenmanagements, in: Prokla. Zeitschrift für kritische Sozialwissenschaft 42: 3, 395-412.

Heires, Marcel/Nölke, Andreas (Hrsg.) 2013: Perspektiven der Finanzialisierung. Ein Handbuch, Wiesbaden, i.E.

Heise, Arne/Lierse, Hanna 2011: The Effects of European Austerity Programmes on Social Security Systems, in: Modern Economy 2: 4, 498-513.

Jessop, Bob 2009: Cultural Political Economy and Critical Policy Studies, in: Critical Policy Studies 3: 3-4, 336-356.

Joerges, Christian 2012: Europas Wirtschaftsverfassung in der Krise, in: Der Staat 51: 3, 357-385.

Kessler, Oliver 2009: Die Subprime-Krise und die Frage nach der Finanzmarktstabilität, in: Zeitschrift für Internationale Beziehungen 16: 1, 161-175.

Kirt, Romain (Hrsg.) 2001: Die Europäische Union und ihre Krisen, Baden-Baden.

Konecny, Martin 2012: Die Herausbildung einer neuen Economic Governance als Strategie zur autoritären Krisenbearbeitung in Europa. Gesellschaftliche Akteure und ihre Strategien, in: Prokla. Zeitschrift für kritische Sozialwissenschaft 42: 3, 377-394.

Lapavitsas, Costas 2012: Crisis in the Eurozone, London.

Lipietz, Alain 1985: Akkumulation, Krisen und Auswege aus der Krise. Einige methodische Überlegungen zum Begriff »Regulation«, in: Prokla. Zeitschrift für kritische Sozialwissenschaft 15: 1, 109-137.

Merkel, Angela 2011: Regierungserklärung von Bundeskanzlerin Merkel zum Europäischen Rat, Deutscher Bundestag, 2.12.2011, http://www.bundesregierung.de/Content/DE/ Regierungserklaerung/2011/2011-12-02-merkel-eu-rat.html; 21.11.2012.

Macartney, Huw 2011: Finance Unravelled: A Historical Materialist Analysis of EU Public Policy, in: Competition \& Change 15: 1, 47-70.

Offe, Claus 2013: Europa in der Falle, in: Blätter für deutsche und internationale Politik 58: 1, 67-80.

Overbeek, Henk 2012: Sovereign Debt Crisis in Euroland: Root Causes and Implications for European Integration, in: The International Spectator 47: 1, 30-48.

Quaglia, Lucia 2012: The Regulatory Response of the European Union: The Case of Hedge Fund Regulation, in: Mayntz, Renate (Hrsg.): Crisis and Control. Institutional Changes in Financial Market Regulation, Frankfurt a.M., 171-195.

Ruggie, John Gerard 1982: International Regimes, Transactions and Change: Embedded Liberalism and the Postwar Economic Order, in: International Organization 36: 2, 379-416.

Scharpf, Fritz W. 2011a: Monetary Union, Fiscal Crisis and the Pre-emption of Democracy (MPIfG Discussion Paper 11/11), Köln.

Scharpf, Fritz W. 2011b: Noch verteidigt Deutschland jeden Meter Boden, in: Süddeutsche Zeitung, 23.12.2011, 12. 
Schelkle, Waltraud 2012: Good Governance in Crisis or a Good Crisis for Governance? A Comparison of the EU and the US, in: Review of International Political Economy 19: 1, 34-58.

Schimmelfennig, Frank 2012: Zwischen Neo- und Postfunktionalismus. Die Integrationstheorien und die Eurokrise, in: Politische Vierteljahresschrift 53: 3, 394-413.

Troost, Axel 2011: Das Kasino bleibt geöffnet. Die Regulierung der Finanzmärkte und was daraus geworden ist, in: Blätter für deutsche und internationale Politik 56: 2, 75-84.

Vaubel, Roland 2012: Die Politische Ökonomie der Staatsschuldenkrise und die Zukunft des Euro, in: Meyer, Dirk (Hrsg.): Die Zukunft der Währungsunion Chancen und Risiken des Euro, Münster, 85-102.

Young, Brigitte 2013: Neoliberale Finanzialisierung und der deutsche Ordoliberalismus in der Euro-Krisenbewältigung, in: Heires, Marcel/Nölke, Andreas (Hrsg.): Perspektiven der Finanzialisierung. Ein Handbuch, Wiesbaden, i.E.

Žižek, Slavoj 2010: A Permanent Economic Emergency, in: New Left Review 64, 85-95. 